\title{
Interactive proteomics: what lies ahead?
}

\author{
Bernhard Suter, Saranya Kittanakom, and Igor Stagljar
}

BioTechniques 44:681-691 (25th Anniversary Issue, April 2008)

doi 10.2144/000112799

\begin{abstract}
Interactive proteomics addresses the physical associations among proteins and establishes global, disease-, and pathway-specific protein interaction networks. The inherent chemical and structural diversity of proteins, their different expression levels, and their distinct subcellular localizations pose unique challenges for the exploration of these networks, necessitating the use of a variety of innovative and ingenious approaches. Consequently, recent years have seen exciting developments in protein interaction mapping and the establishment of very large interaction networks, especially in model organisms. In the near future, attention will shift to the establishment of interaction networks in humans and their application in drug discovery and understanding of diseases. In this review, we present an impressive toolbox of different technologies that we expect to be crucial for interactive proteomics in the coming years..
\end{abstract}

\section{INTRODUCTION}

Proteins are the basic building components of the cell that carry out diverse functions such as catalyzing chemical reactions as enzymes, binding other molecules as receptors, and forming structural components. Physical associations between different proteins, so-called protein-protein interactions (PPIs), are an essential aspect in all biological pathways and signaling mechanisms, and are strongly predictive of functional relationships. Mapping of PPI networks is particularly useful for the functional annotation of the large number of newly identified open reading frames (ORFs) that were identified in the recent sequencing of the human genome and the genomes of various model organisms. The exploration of PPIs on a large-scale or global level is referred to as interactive proteomics, cell-map, or interaction proteomics $(1,2)$. Analysis of protein assemblies can also be considered as one part in the wider context of structural proteomics, which is the systematic analysis of protein structure and folding $(3,4)$. Establishing networks of PPIs will eventually lead to a better understanding of cellular pathways and functional associations and allow a more holistic view of the cell, as opposed to the classical one-protein-at-a-time approach.
Indeed, the recent exploration of entire interactomes from different organisms was a consequence of the development of technologies that allowed the assessment of PPIs on a high-throughput (HTP) level. In this review, we begin with the current state of interactive proteomics research, describing established mature technologies like yeast two-hybrid ( $\mathrm{Y} 2 \mathrm{H})$ and affinity purification-mass spectrometry (AP-MS), which have been applied for genome-wide or pathway-specific screens in different model organisms and in human cells. We then present emerging technologies, including in vivo methodologies $(\mathrm{Y} 2 \mathrm{H}$ derivatives, protein fragment complementation in mammalian cells, detection of PPIs by fluorescence and bioluminescence) and biochemical approaches (MS-based methods, largescale analysis of tagged arrays). We discuss these methodologies in the context of future directions in interactive proteomics, considering the extension of large-scale approaches into mammalian organisms, and the application of interactive proteomics for drug discovery and research on human diseases. Unfortunately, space restrictions leave us unable to discuss technologies that are currently used to explore PPIs in vitro. This includes the protein-chip technology that has emerged as a powerful tool for the parallel analysis of protein interactions and biochemical activities (5,6). Likewise, we refer the reader elsewhere for excellent reviews on protein engineering and phage display (7-9). We will also not discuss data interpretation and the analysis of network topologies that are addressed by bioinformatics $(10,11)$.

\section{GENETIC METHODS FOR THE ANALYSIS OF PROTEIN INTERACTIONS}

\section{Yeast Two-hybrid Assay}

In the time since Song and Fields devised their original interaction trap assay in 1989 (12), the Y2H system has become the most widely used method to assess both individual PPIs and entire interactomes. $\mathrm{Y} 2 \mathrm{H}$ is very cost effective, convenient to use, and easily adaptable for HTP screening procedures. Exploiting the modularity of eukaryotic transcription factors, a known protein (bait) protein is fused to the DNA-binding domain (DBD), and an interacting protein (prey) is fused to the activation domain (AD) of a transcriptional activator (Figure 1A). A physical interaction between bait and prey leads to the activation of a reporter gene construct that serves as a selection marker. Using the yeast transcription machinery as a vehicle, physical interactions between 
proteins from any other organism can be easily monitored. However, a major drawback in $\mathrm{Y} 2 \mathrm{H}$ is false-positive interactions that occur upon self-activation of reporter genes by individual bait and prey proteins. Furthermore, since interactions between all bait and prey proteins are confined in the nucleus of a lower eukaryote, PPI analysis by Y2H suffers from a lack of contextual specificity. Consequently, a variety of methods have been developed to improve the $\mathrm{Y} 2 \mathrm{H}$ system and adapt it for different purposes. For more reading on $\mathrm{Y} 2 \mathrm{H}$, we refer to the article of Ratushny and Golemis in this issue of BioTechniques (p. 655) and other reviews (13-15).

Several years ago, two different laboratories generated a comprehensive overview of the yeast Saccharomyces cerevisiae proteome by $\mathrm{Y} 2 \mathrm{H}$, using matrixes with all 6000 yeast ORFs cloned into bait and prey vectors $(16,17)$. Since then, Y2H screens have been done for the metazoan model organisms Drosophila melanogaster and Caenorhabditis elegans $(18,19)$ and, more recently, for Homo sapiens $(20,21)$. Because of the sheer size and complexity of the mammalian proteomes, human Y2H screens are more practical on a subgenome scale and have been centered upon specific signaling pathways or disease factors (22-24). In one of these studies, $\mathrm{Y} 2 \mathrm{H}$ screens were performed with bait proteins that are involved in several known inherited ataxias (23). Inherited ataxias are neurodegenerative disorders with common clinical and pathological features. The molecular basis of these diseases, however, is not well defined. Interestingly, Y2H screens revealed that many ataxia-associated proteins share interacting partners, suggesting that these proteins are functionally connected and act in a concerted manner. This work provides an excellent understanding of how interactome studies using $\mathrm{Y} 2 \mathrm{H}$ can help us understand common pathogenic mechanisms and identify candidate genes for complex human diseases.

As such, $\mathrm{Y} 2 \mathrm{H}$ is still the method of choice for researchers who conduct human interactome studies, although one would expect that screening human proteins in mammalian cells would be more context-specific. Indeed, the principles of $\mathrm{Y} 2 \mathrm{H}$ have been implemented in several versions of mammalian twohybrid approaches (25-27). Application of the large-scale screening procedures is inherently more difficult, however, for mammalian cells than for yeast. This has mainly to do with the ease with which yeast can be manipulated, grown, and maintained. Therefore, the use of mammalian two-hybrid and its variants is currently limited to those cases that can only be addressed within the environment of the mammalian cell.

\section{Yeast Two-hybrid in Drug Discovery}

The large number of PPIs being identified by the combined efforts in interactive proteomics studies promises
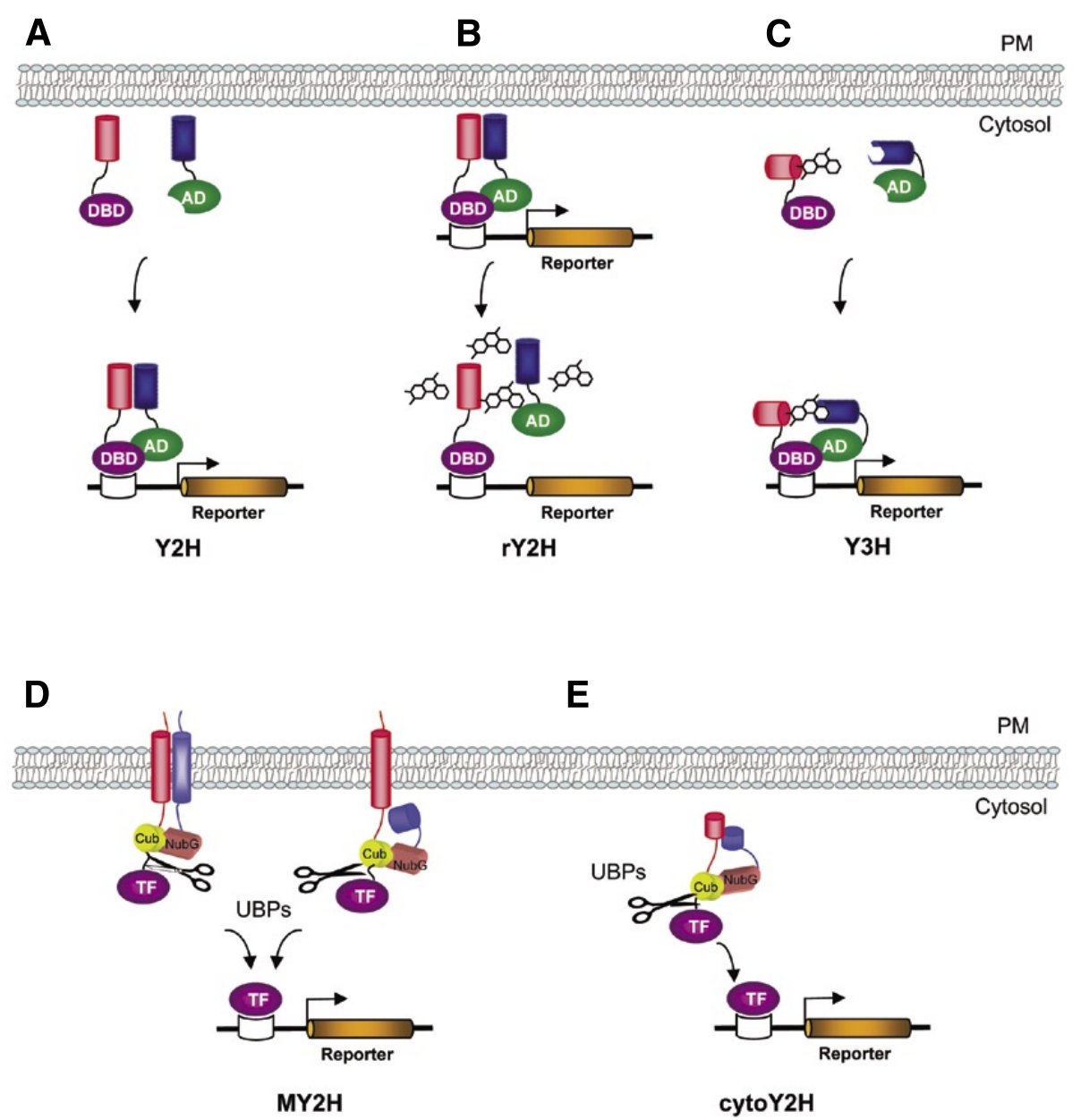

Figure 1. Applications of the yeast two-hybrid system. (A) Classical yeast two-hybrid (Y2H). A bait protein of interest (red) is fused to the DNA-binding domain (DBD) of a transcriptional activator, whereas a prey protein or a library of prey proteins (blue) is fused to the activation domain (AD). A typical transcription factor in $\mathrm{Y} 2 \mathrm{H}$ is the Gal4 activator protein. The physical interaction between bait and prey reconstitutes the transcription factor (TF), resulting in the expression of the reporter gene, thereby enabling the yeast cell to grow on selective medium. (B) Reverse yeast two-hybrid for drug screening $(\mathrm{rY} 2 \mathrm{H})$. Reconstitution of the transcription factor by bait-prey interaction drives a counterselectable reporter that generates a toxic metabolite and leads to cell death. A compound that interferes with the bait-prey protein interaction prevents the reconstitution of the transcription factor and rescues viability. (C) The yeast three-hybrid system (Y3H) for small molecule screening. The bait consists of a DBD fused to a protein scaffold that binds a defined small molecule with high affinity. The prey consists of an $\mathrm{AD}$ that is fused to a cDNA library. The yeasts are grown in the presence of a hybrid molecule that consists of a scaffold-binding molecule and a compound of interest. If a prey protein binds the compound of interest, a functional transcription factor is reconstituted and a reporter gene is activated. (D) Membrane yeast two-hybrid system (MY2H). In MY2H, integral or peripheral membrane proteins (baits) are fused to the C-terminal half of ubiquitin (Cub), followed by a transcription factor (TF). Preys (membrane or cytosolic proteins) are expressed as fusions with the $\mathrm{N}$-terminal half of ubiquitin (Nub). Bait-prey interaction reconstitutes native ubiquitin, which is then cleaved by endogenous ubiquitin specific protease (UBPs, black scissors). The transcription factor enters the nucleus and activates reporter gene expression. (E) Cytosolic yeast two-hybrid system (cytoY2H). This system is also based on principles of the splitubiquitin assay but detects protein-protein interactions in the cytoplasm. 
to reveal a vast array of novel, therapeutic targets. The success of the $\mathrm{Y} 2 \mathrm{H}$ in the detection of PPIs motivated adaptations of the system to assess interaction of proteins with small molecules in a physiological setting. The use of these $\mathrm{Y} 2 \mathrm{H}$ approaches has been somewhat limited, however, since yeast cells are generally not very permeable to small molecules. In one successful approach, Y2H was undertaken to identify compounds that inhibit the association of the c-Myc oncoprotein with its interaction partner Max. This study facilitated the identification of a series of compounds that exhibited specificity against c-Myc/Max function in mammalian cells and inhibited tumor growth in vivo (28). Another study considered the binding of the Ras oncoprotein to its downstream effector Raf-1 (29). Using a modified, subtractive $\mathrm{Y} 2 \mathrm{H}$ approach, a library of 73,400 compounds was screened for small molecules that inhibit the interaction of Ras with Raf-1. Several candidate molecules were identified and a reversion of Ras-transformed phenotypes was confirmed for three of them.

The reverse yeast two-hybrid system (rY2H) uses a counterselection strategy to generate a positive readout for disruption of the designated PPIs (Figure 1B) $(30,31)$. Therefore, parallel toxicity testing of the compounds is not required in the screening procedure. Commonly, the yeast $U R A 3$ gene is used as a counterselection marker, since it converts 5fluoroorotic acid (5-FOA) into a toxic metabolite that leads to cell death. A small molecule that inhibits bait-prey interaction is expected to rescue viability in the presence of 5-FOA. An application of this screening scheme revealed that the interaction between the activin receptor $\mathrm{R} 1$ and the immunophilin protein FKBP12 is disrupted by the compound FK506 (30).

Another adaptation of $\mathrm{Y} 2 \mathrm{H}$ for drug screening is the yeast three-hybrid $(\mathrm{Y} 3 \mathrm{H}$; Figure 1C). $\mathrm{Y} 3 \mathrm{H}$ is a forward approach to screen for prey proteins that interact with a small molecule displayed by the bait, and not with the bait protein itself $(32,33)$. A DBD is fused to a protein domain that binds a known compound with high affinity. This compound is part of a hybrid molecule, which has another part that binds to potential prey proteins fused to the $\mathrm{AD}$. In one version, the $\mathrm{DBD}$ was expressed as a fusion protein with dihydrofolate reductase (DHFR) (33), which binds methotrexate (MTX) with high affinity. The DHFR-MTX system was applied in a large-scale approach to explore human targets of kinase inhibitors (34). Various known cyclin-dependent kinase (CDK) inhibitors were displayed in the form of MTX hybrid molecules to screen for interacting prey proteins. Known cell cycle CDKs, novel candidate CDK-like, and/or CDK-unrelated kinase targets could be identified. Many of these interactors were confirmed by independent methods. These results demonstrate that $\mathrm{Y} 3 \mathrm{H}$ is a valuable tool in the discovery of lead compounds.

\section{Membrane Yeast Two-hybrid Assay}

Nearly one-third of all genes in various organisms encode integral membrane and/or membrane-associated proteins that are not amenable for analysis by $\mathrm{Y} 2 \mathrm{H}$ due to their hydrophobic nature and nonnuclear localization. Our laboratory previously developed the membrane yeast two-hybrid (MY2H) system (35), which allows the identification of PPIs of membrane bait proteins with either membrane or cytosolic preys (Figure 1D). MY2H is an adaptation of the splitubiquitin assay that reconstitutes the two halves of ubiquitin upon interaction of bait and prey proteins (36). Upon cleavage of the reconstituted ubiquitin by ubiquitinspecific proteases, a transcription factor is released and activates reporter gene expression in the nucleus. MY2H has been adapted for use in a matrix-based screening assay for interactors of 705 selected yeast integral membrane proteins and has identified 1985 putative interactions involving 536 proteins (37). More recently, a modified version of MY2H called integrative MY2H assay $(i \mathrm{MY} 2 \mathrm{H})$ has been developed and used in the identification of six novel interacting partners of the yeast $\mathrm{ABC}$ transporter Ycflp (38). MY2H has also been used to successfully identify interactors for mammalian transmembrane proteins, which have been confirmed using other methods $(39,40)$. In one example, three new interactors were identified for the human epidermal growth factor receptor ErbB3 (40). Our laboratory is currently performing comprehensive MY2H screenings to map the interaction partners of numerous yeast and human integral membrane proteins (membrane protein interactome). Since transmembrane proteins like ion channels, G-protein-coupled receptors (GPCRs), and transporters constitute major classes of drug targets, MY2H has the potential to be a valuable tool for drug discovery and toxicology. We are currently developing novel approaches to address potential changes in interaction patterns upon drug action or ligand binding and believe that these innovations combined with improvements in the experimental strategy will fulfill the promise of this methodology within in the next five years (S. Kittanakom, M. Chuk, J. Snider, M. Rehal, B. Suter, J. Curak, and I. Stagljar, unpublished data).

The cytosolic yeast two-hybrid system (cytoY2H, Figure 1E) is a variant of MY2H in which baits and preys interact in the cytoplasm (41). CytoY2H can be applied for proteins that are difficult to study in the nuclear context, including transcription factors that may self-activate reporter gene expression. The cytoY2H system was used to screen a cDNA library and identify several new interaction partners of Urilp, a previously uncharacterized yeast protein.

\section{Protein Fragment \\ Complementation Assays}

In protein fragment complementation assays (PCA), a PPI is detected by the reconstitution of a functional reporter protein from two split nonfunctional halves (Figure 2A). Notably, PCA relies on the proper refolding of rationally dissected fragments and not on the docking of preformed subunits. In principle, splitubiquitin can be considered as an early application of PCA (36). PCA fragments are designed in a way that prevents spontaneous refolding. In its original implementation, PCA is based on protein interaction-induced folding and reconstitution of the enzymatic activity of DHFR $(42,43)$. Reconstitution of functional DHFR was demonstrated in vivo by selection on trimethoprim and in vitro by DHFR activity assays. A screening scheme was then devised for DHFR-PCA to detect PPIs in two different signaling pathways (44). These were two pathways that control initiation of translation, the insulin and growth factor receptor tyrosine kinase (RTK)-mediated pathway, and a parallel pathway directed by the FK506 
A

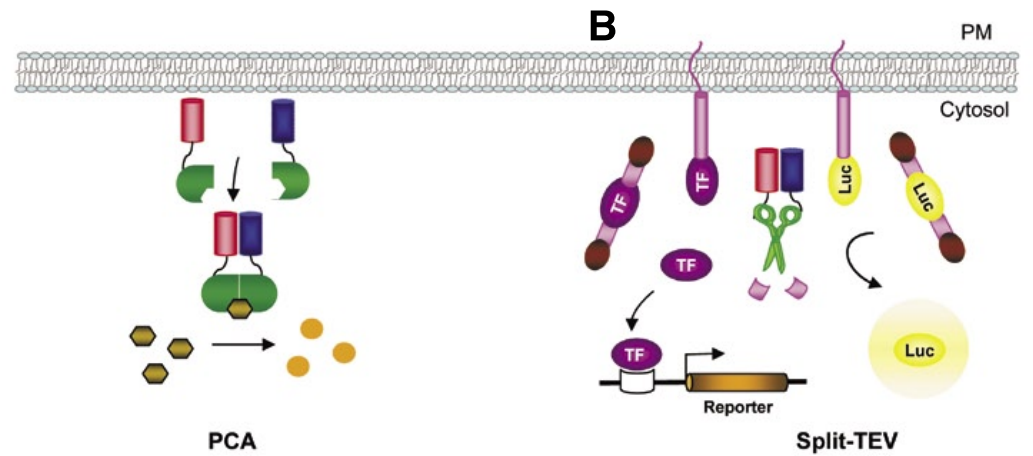

C

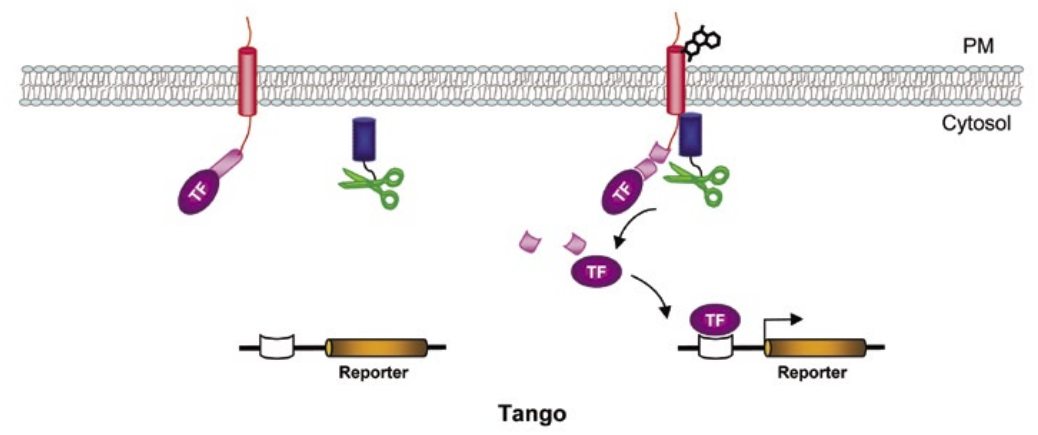

D

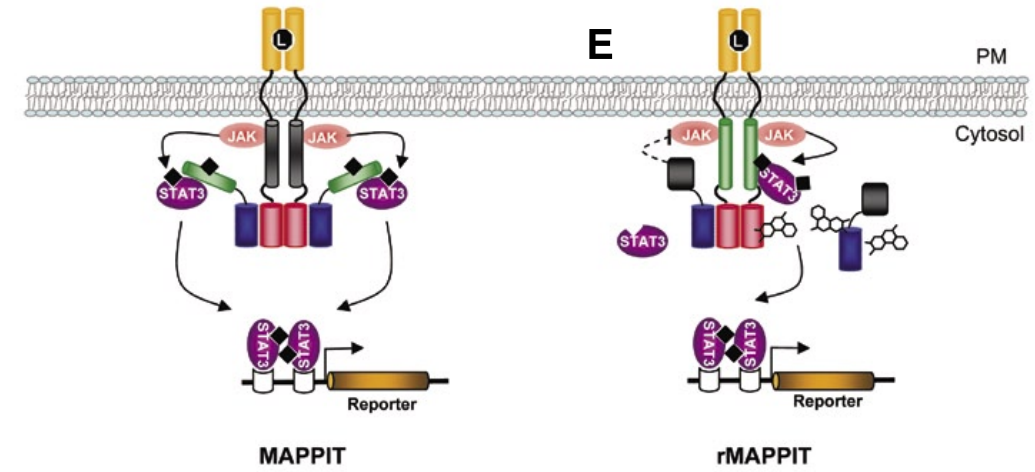

Figure 2. Complementation and reconstitution systems in mammalian cells. (A) Protein fragment complementation assay (PCA). A reporter enzyme (green) is rationally dissected into complementary fragments that are fused with bait (red) and prey (blue) proteins. Bait-prey interaction results in the correct refolding of the enzyme and in the restoration of enzyme activity. (B) Split-TEV system. The tobacco etch virus (TEV, green scissors) protease is functionally reconstituted by a bait-prey interaction. TEV cleavage of the recognition sequence (pink) releases a transcription factor (TF) that was either sequestered in the membrane or in the cytosol. The transcription factor enters the nucleus and drives reporter gene expression (transcription-coupled split-TEV). Alternatively, cleavage by TEV releases a luciferase (Luc), which is only active when liberated (proteolysis-only split-TEV). (C) Tango method to monitor receptor-ligand interactions. A transcription factor is tethered to a membrane-bound receptor with a linker that contains a cleavage site (pink) for a specific protease (TEV, green scissors). A signaling protein that binds only to activated receptors is fused to the TEV protease. Receptor activation by ligand binding results in the release of the transcription factor and the activation of a reporter gene in the nucleus. (D) Mammalian protein-protein interaction trap (MAPPIT). A bait protein is fused with the transmembrane and intracellular part of a cytokine receptor variant (gray) that cannot recruit the STAT3 transcription factor. Recruitment and activation of STAT3 by Janus kinases (JAK) normally occurs when the receptor is activated and clustered by ligand (L) binding to the extracellular domain (gold) of a native or a chimeric receptor. Prey proteins are fused to a functional intracellular part of the receptor that contains docking sites for STAT3 (green). Interaction between the bait and prey proteins therefore complements the signaling function of the receptor. Phosphorylation (black diamond) of these docking sites by the activated JAK kinases leads to the recruitment and phosphorylation of STAT3. Induced (phosphorylated) STAT3 transcription factor translocates to the nucleus and activates a STAT3-responsive luciferase reporter. (E) Reverse MAPPIT (rMAPPIT). Bait protein is linked to a functional cytokine receptor with docking sites for STAT3. Recruitment of a prey protein fused to an inhibitory domain (gray) results in suppression of signaling via a functional leptin chimeric bait receptor. Disruption of bait-prey interaction by compound (or competitor protein) results in restoration of signaling and reporter gene induction. binding-rapamycin-associating protein (FRAP). Results revealed considerable cross-talk between the RTK and the FRAP pathways and detectable effects of drugs that target their components.

Different applications have been devised for PCA, and a variety of reporter proteins were used in the procedure (see Reference 45 for detailed review). The readouts include survival selection, reporter gene activation, and reconstitution of luminescence or fluorescence. Furthermore, PCA can be applied directly in every organism and cell type of interest. The method has matured considerably and holds a great potential in drug discovery and protein engineering (45).

\section{Split-TEV Assay}

Split-TEV is a novel approach to monitor constitutive and regulated PPIs in mammalian cells by fragment complementation of the tobacco etch virus (TEV) protease (46). Inactive fragments from a dissected TEV protease refold and regain activity when brought into proximity by the interaction between proteins to which the fragments are fused (Figure 2B). Reconstituted TEV cleaves TEV-recognition sequences in fusion protein constructs that sequester reporter proteins. The reporter protein is either a transcription factor in the transcriptioncoupled system or the light-emitting enzyme luciferase in the proteolysis-only variant. The reporter proteins are either sequestered to the cell membrane or the cytoplasm. In its first application, splitTEV was used to monitor Erb2/Erb4 receptor tyrosine kinase heterodimerization induced by neuregulin (46). In principle, split-TEV is a PCA variant since refolding a functional enzyme depends on the PPI between bait and prey. However, TEV does not function as a direct reporter, but activates a reporter protein in an irreversible manner, thus generating a more stable readout for the TEV reconstitution. The flexibility and the modular design of the system could make it a good choice for future largescale approaches.

\section{Tango Assay}

Tango is an innovative, recently developed methodology that exploits the binding of activated receptors by known 
signaling proteins to monitor receptor activation and ligand binding (47). An activated receptor recruits a signaling protein fused to the TEV protease (Figure 2C). Cleavage by TEV releases a transcription factor that is attached to the receptor. The irreversible release of the transcription factor converts the transient signaling event into stable transcription of a reporter gene. Tango relies solely on exogenous genes introduced into the cell and therefore avoids interference from endogenous signaling pathways. The Tango assay was modified to monitor the activity of three different classes of receptors: $G$ protein-coupled receptors (GPCRs), receptor tyrosine kinases, and steroid hormone receptors. The Tango method was successfully used to identify a ligand for the orphan receptor GPR1 (47). In the current form, Tango is a system to monitor receptor-ligand interactions. In the future, it may be adapted to screen for novel signaling proteins that bind to activated receptors.

\section{Mammalian Protein-Protein Interaction Trap}

Mammalian protein-protein interaction trap (MAPPIT) is an elegant system in which the interaction between a bait and prey protein restores liganddependent cytokine receptor signaling (48) (Figure 2D). MAPPIT relies on the unique properties of the JAK-STAT (Janus kinase/signal transducer and activator of transcription) signaling pathway, which is mediated by type I cytokine receptors (49). MAPPIT is not a PCA application, but rather can be designated as a two-hybrid complementation system. MAPPIT is especially applicable to the analysis of signal transduction pathways, since the PPIs are detected in their normal physiological context in the cytoplasm. The readout exploits the nuclear translocation capability of activated STATs, and therefore the PPIs are not forced into the artificial context of the nucleus. In the first application of MAPPIT in cDNA screening, cytokineinducible $\mathrm{SH} 2$-containing protein (CIS) and suppressor of cytokine signaling-2 (SOCS-2) were identified as interactors of a phosphotyrosine-binding motif in the erythropoietin receptor (48). A variety of other screens have been undertaken to identify interaction partners for various binding motifs (50-52). Hence, MAPPIT provides a useful tool for the analysis of physiologically relevant, inducible protein interactions.

The reverse version of MAPPIT (rMAPPIT) generates a positive readout upon disruption of a PPI (53). In rMAPPIT, the prey recruits a protein that inhibits receptor activity and STAT recruitment (Figure 2E). The disruption of the bait-prey interaction activates the receptor and results in transcription by STATs. This scheme was used to monitor drug effects on three different PPIs. Most recently, a three-hybrid version of the system, the so-called mammalian small molecule protein interaction trap (MASPIT), was generated (54). As in Y3H, DHFR is fused to the receptor-bait, and displays a hybrid ligand consisting of MTX and the compound of interest. MASPIT was also adapted to screen a human cDNA for preys that specifically bind to known kinase inhibitors for $\mathrm{Abl}$ and Src kinases. A variety of kinases were identified that were previously not known to be targets of the compounds (54).

\section{DIRECT VISUALIZATION OF PROTEIN INTERACTIONS IN VIVO}

\section{Fluorescence/Bioluminescence Resonance Energy Transfer (FRET/BRET) Assays}

Fluorescence resonance energy transfer (FRET) using variants of the green fluorescent protein (GFP) has become a very important application for the characterization of PPIs in living cells (55). FRET is a direct, radiationless energy transfer between two spectrumoverlapped fluorophores: the donor (CFP, cyan; BFP, blue) and the acceptor (variant GFP, green; YFP, yellow) molecules (Figure 3A). The bait protein of interest is linked to the donor whereas the prey protein is linked to the acceptor. An interaction between bait and prey brings the donor and acceptor fluorophores into close proximity with one another, resulting in FRET and emission of fluorescence from the acceptor. The technique is ideally suited to assess the real-time dynamics of complex formation and dissociation in vivo. Furthermore, the subcellular compartment where an interaction takes place can be readily determined. A drawback of standard FRET methods is that they often suffer from fluorescent background or photobleaching. Bioluminescence resonance energy transfer (BRET) was developed to avoid these problems by replacing the donor fluorophore with luciferase from Renilla reniformis $(56,57)$.

BRET is especially applicable to studying the signaling and dynamics of receptor proteins, including GPCRs $(58,59)$. In GPCR signaling, $\beta$-arrestin is recruited after activation of the receptor by compounds that function as receptor agonists. Conversely, antagonists block the signaling by agonists and $\beta$-arrestin recruitment. Recruitment of $\beta$-arrestin can be adapted for a screening scheme for GPCR agonists and antagonists using BRET (60). In a screen for antagonists of the chemokine receptor CCR5, about 26,000 compounds were examined for inhibition of agonistpromoted $\beta$-arrestin recruitment (61). Of these, twelve compounds were identified that inhibited signaling by CCR5. Three of the potential hits were further tested using other functional assays, and their inhibitory role on agonist-stimulated GPCR signaling was confirmed. BRET has also been adapted for imaging the dynamics of a receptor- $\beta$-arrestin association at the single-cell level in real time (62). The BRET- $\beta$-arrestin scheme can be understood as an example of how a pharmacologically relevant PPI can be applied for drug discovery in HTP screens.

\section{Bimolecular Fragment Complementation}

In bimolecular fluorescence complementation (BiFC), a PPI induces the complementation of a fluorescent protein from two dissected halves (Figure 3B). $\mathrm{BiFC}$ is therefore an adaptation of PCA. In an early application, BiFC between two nonfluorescent fragments of the yellow fluorescent protein (YFP) was used to investigate interactions among the bZIP and Rel family transcription factors (63). Fragments of fluorescent proteins with different spectral characteristics allow the simultaneous visualization of 
interactions between different proteins in the same cell (multicolor BiFC) (64). A large number of PPIs have been characterized and numerous applications of the methods have been reported (65). BiFC has also been used in conjunction with cDNA libraries to screen for PPIs (66).

BiFC reconstitutes a fluorescent protein and is therefore, in principle, more sensitive than FRET, which changes existing fluorescence signals. On the other hand, FRET allows association-dissociation analysis of PPIs, whereas in BiFC, the process of protein folding interferes with the dynamics of these processes. Recently, fragment complementation has also been adopted for luciferases. Folding of Gaussia and Renilla luciferases is reversible and therefore allows the dissociation of protein complexes to be monitored $(67,68)$. However, activity of luciferase is dependent on the addition of the exogenous substrate luciferin, whereas BiFC is solely dependent on excitatory fluorescent light. Choosing a method for in vivo analysis of PPIs depends upon the specific requirements that each experiment presents in terms of sensitivity and dynamics.

Most intriguingly, BiFC, BRET, and a modified mammalian two-hybrid system have been applied for the detection of PPIs in living animals using sensitive molecular imaging technologies $(69,70)$. These approaches could be a first step to real in vivo proteomics and provide us with the possibility to assess drug-specific effects on PPIs in the context of the whole organism.

\section{Proximity Ligation In Situ Assay (P-LISA)}

Approaches to assess PPIs in vivo generally involve genetic manipulation of the targeted proteins. Baits and preys that are fused to tags may become destabilized and/or mislocalized, thereby complicating their analysis in the natural context. Recently, an innovative study by Ulf Landegren and co-workers adapted the proximity ligation technology to perform high-resolution analysis of PPIs in situ (P-LISA; Figure 3C) (71,72). Proximity probes are oligonucleotides attached to antibodies that bind proximal targets (73). In P-LISA, two (or more) interacting proteins that are targeted by proximity probes guide the formation of circular DNA strands (72). The DNA circles, in turn, serve as templates for localized rolling-circle amplification (RCA), which results in approximately 1000-fold amplification of a complementary ssDNA product that can be detected using fluorescent probes. P-LISA was successfully used to detect the association between c-Myc and Max. The group could also demonstrate the disruption of the c-Myc/Max complex by an experimental compound that was previously identified by $\mathrm{Y} 2 \mathrm{H}$ ((28), see also section on yeast two-hybrid in drug discovery). Notably, P-LISA is not a real in vivo method, since the cells need to be fixed and permeabilized. On the other hand, in P-LISA the expression of target proteins is not affected or perturbed by genetic manipulation. Most importantly, the method allows the localized detection of interacting proteins down to the single-molecule level. Therefore, P-LISA occupies a unique niche and has great potential for use in interaction proteomics.

\section{BIOCHEMICAL ANALYSIS OF PROTEIN INTERACTOMES}

\section{Affinity Purification-Mass Spectrometry Approach}

Biochemical isolation of protein complexes by affinity purification (AP) with subsequent identification of the purified complex components by mass spectrometry (MS) was successfully used for global interactome analysis in the yeast S. cerevisiae (74-77). AP-MS identifies multimeric protein assemblies, but does not indicate the precise physical associations within them. The readout is therefore distinct from that of the methodologies discussed in this review (e.g., Y2H, BiFC, PCA) that detect binary interaction pairs.

The tandem affinity (TAP) protein purification scheme is the most widely used AP-MS approach (Figure 4A). It allows for a two-step purification of tagged protein complexes under physiological conditions $(78,79)$. Two groups used the TAP procedure in comprehensive surveys of the entire yeast proteome, in which all ORFs were tagged. These studies identified binding partners for a large fraction of the purified proteins and revealed that the yeast proteome can be partitioned into approximately 500 large protein assemblies (75-77). More recently, the TAP approach has also been used to assess PPIs in signaling pathways in Drosophila melanogaster (80) and in human cells $(81,82)$. One study addressed a signaling pathway that is triggered by the proinflammatory cytokine tumor necrosis factor TNF- $\alpha$ and leads to the activation of the transcription factor NF$\kappa \mathrm{B}$ (81). Thirty-two components of the pathway were TAP-tagged and purified. In total, 221 interactions were identified, of which 10 were found to constitute new modulators of the pathway. The roles for these novel modulators in the TNF- $\alpha / N F-\kappa B$ pathway were confirmed by the effect of small interfering RNA (RNAi)-mediated knockdown on NF- $\mathrm{BB}$ dependent luciferase expression.

The classical TAP-tag construct has limitations for its application in mammalian cells, mostly related to the low protein yields attained. Therefore, it is important to develop improvements and efficient procedures for AP-MS in mammalian cells. A modified TAP-tag that contains a protein $G$ tandem repeat and the streptavidin-binding peptide (GS-TAP) results in a 10-fold increase in protein-complex yield when compared with the conventional TAP-tag (83). Purifying the Ku70-Ku80 DNA-repair complex resulted in the successful isolation of both known complex components and a number of other proteins. In another study, a short TAP-tag consisting of a FLAG and a $6 \times$ HIS tag was used to purify the Pax7 transcription factor from satellite cells in skeletal muscle (84). Pax7 was found to associate with a histone methyltransferase (HMT) complex that stimulates transcriptional activation of target genes to regulate entry into myogenic development. Currently, a large-scale approach is under way to tag about 200 oncogenes and other important disease-linked factors with the FLAG$6 \times$ HIS tag in murine stem cells by transfection and "knock-in" by homologous recombination (A. Emili, J.F. Greenblatt, J. Rossant, personal communication). Transgenic mice expressing these tagged oncogenes will be used to address differential interaction patterns in various tissues. Moreover, a genome-wide collection of lentiviral expression vectors for the tandem affinity tagging of all human ORFs is currently being devised 

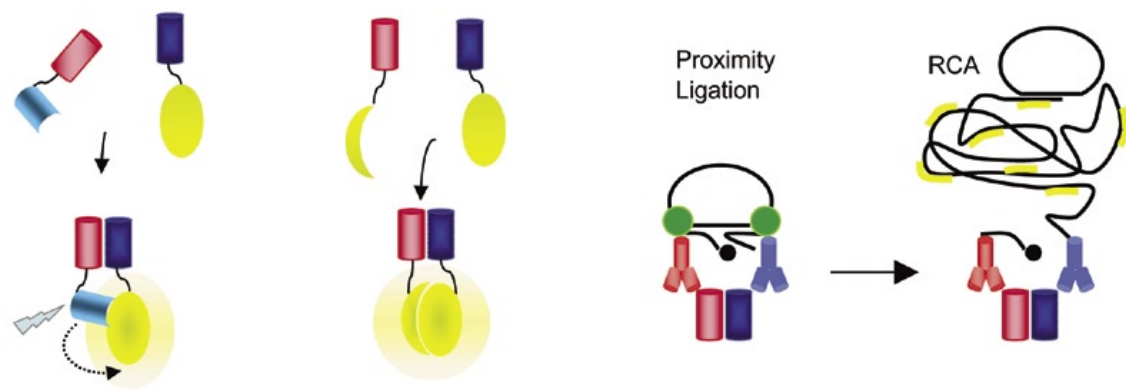

FRET/BRET

BiFC malian interactome mapping), an automated HTP technology for the systematic mapping of PPI networks in mammalian cells (Figure 4C) (87). LUMIER was applied to the pathway that is induced by transforming growth factor- $\beta$ (TGF $\beta$ ). Screening for PPIs was done by co-precipitating core components of the TGF $\beta$ pathway that were fused to the Renilla luciferase enzyme with $5183 \times$ FLAG-tagged cDNA encoded proteins. LUMIER detected pathway-specific interactions that were either constitutive or dependent on TGF $\beta$-signaling and revealed critical connections to other signaling networks, such as the p21-activated kinase (PAK) and occludin, a protein that is associated with tight junctions and cell polarity. Further application of LUMIER to other signaling pathways will undoubtedly reveal the full potential of this approach in mammalian proteomics.

\section{Quantitative Immunoprecipitation Combined with Knockdown}

Proteins that co-purify nonspecifically with a targeted protein complex often complicate identification of the interaction partners by MS. The laboratory of Matthias Mann developed the so-called QUICK (quantitative immunoprecipitation combined with knockdown) methodology, which is ideally suited to circumvent this problem (Figure 4D) (88). The stable isotopic amino acids in cell culture labeling (SILAC) (89) approach was used to differentially label proteins in two different cell cultures. In one culture (control), the target protein was knocked down with a RNAi. After protein extraction, the lysates were mixed in equal parts and immunoprecipitated with a specific antibody for the target protein. The differentially labeled proteins in the precipitate were then identified by MS. Proteins that display both isotopes can then be assigned to the unspecific background, whereas proteins that are not labeled with the isotope from the control culture represent true associations with the target protein. QUICK circumvents protein tagging and addresses the dynamics of PPIs in an undisturbed cellular environment. On the other hand, the QUICK methodology requires specific antibodies for every target protein and schemes for isotope

\section{Luminescence-based Mammalian Interactome Mapping}

The Wrana laboratory developed LUMIER (luminescence-based mam- a simple one-step pulldown approach in

$S$. cerevisiae that can be used to confirm 


\section{Review}

A

$$
\text { Yeast TAP }
$$

Mammalian TAP
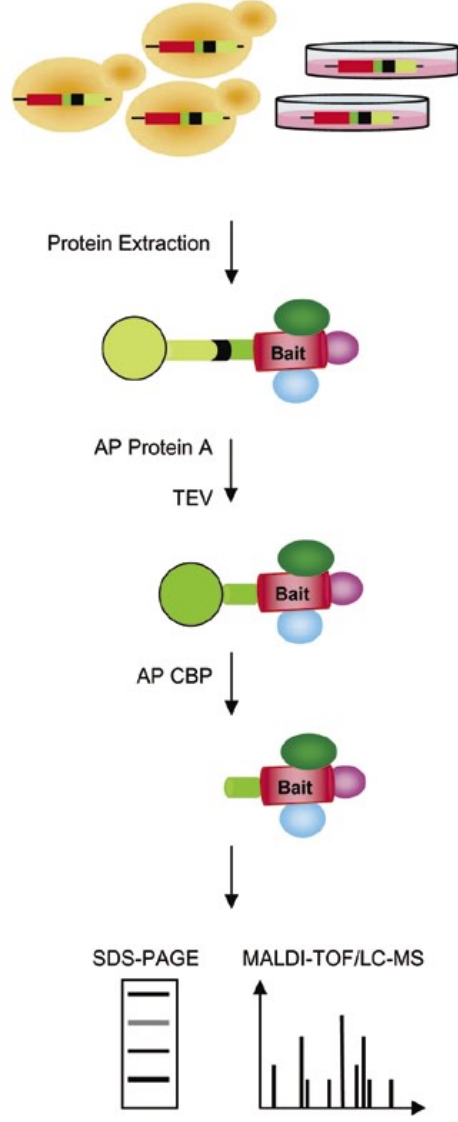

TAP-MS
B

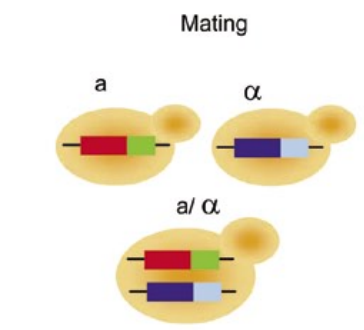

Protein Extraction

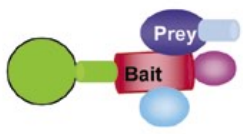

Pull-Down

$6 \times$ HIS

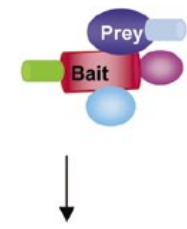

Western Blot

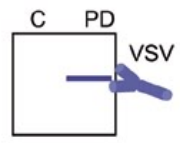

Cross-and-Capture
C

Cotransfection
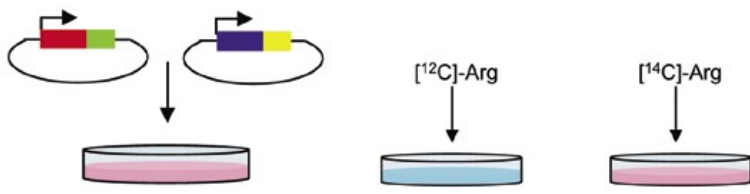

+RNAi

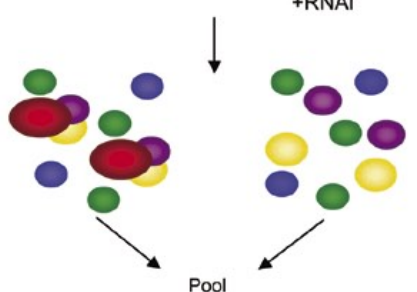

Pool

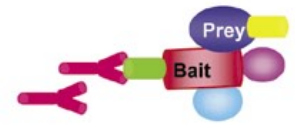

Immunoprecipitation
Anti-FLAG

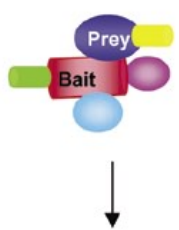

Luminescence in HTP Assay

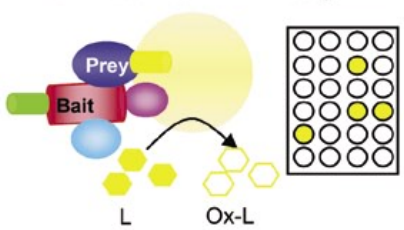

LUMIER

QUICK

Figure 4. Biochemical methods for interactome mapping. (A) Tandem affinity procedure (TAP). The TAP-tag contains two separate moieties (protein A and a calmodulin-binding protein, CBP, in the classical approach) separated by a recognition sequence for the tobacco etch virus (TEV) protease. The TAP sequence is fused to the $\mathrm{C}$-terminus (or $\mathrm{N}$-terminus) of a coding sequence. Protein extracts from yeast or mammalian cells expressing the TAP-tagged protein (red) are subjected to two sequential affinity purification (AP) steps (IgG for protein A, light green; calmodulin for CBP, dark green) and cleavage by the TEV protease. Purified protein complexes are separated by denaturing gel electrophoresis. Separated proteins are digested with trypsin into small peptides that can be identified by mass spectrometry. The identity of the proteins is determined by database searches. (B) Cross-and-Capture. Yeast open reading frames (ORFs) are either tagged as baits with six histidines $(6 \times$ HIS) in the a-haplotype or as preys with a triple VSV-tag (VSV) in the $\alpha$-haplotype. Mating of a and $\alpha$ haploid strains results in diploid cells that express baits and preys. From these diploids, baits and bait-associated prey proteins are extracted and isolated with nickel beads. Isolated proteins are then separated by gel electrophoresis and probed by Western blot analysis. The interaction between bait and prey proteins is confirmed by the presence of the VSV-tag in the isolated pulldown fraction. (C) Luminescence-based mammalian interactome mapping (LUMIER). Prey proteins fused to the Renilla luciferase enzyme and individual bait proteins tagged with the FLAG sequence are coexpressed in mammalian cell cultures. Cell extracts are treated with anti-FLAG antibodies for immunoprecipitation. Light emission upon addition of luciferin, the substrate for luciferase, displays a bait-prey interaction in the pulldown. LUMIER is an automated screening procedure. (D) Quantitative immunoprecipitation combined with knockdown (QUICK). Proteins in tissue cultures (turquoise, rose) that endogenously express the target protein of interest are labeled with light $\left[{ }^{12} \mathrm{C}\right]$-arginine or heavy $\left[{ }^{14} \mathrm{C}\right]$-arginine isotopes. In one culture (control), the target protein (red) is depleted by a small interfering RNA (RNAi). The lysates from the tissue cultures are combined and immunoprecipitated. The target-specific antibody not only precipitates proteins that are in a complex with the target (yellow, purple), but also with a number of unspecific contaminants (blue, green). The differentially labeled proteins are then separated (liquid chromatography, LC) and identified by tandem mass spectrometry (MS). Proteins that specifically associate with the target display only the light isotope, whereas proteins that display both light and heavy isotopes do not interact with the target. 
labeling and MS. However, QUICK could be more easily adapted for large-scale screening in the future, once interfering RNA and antibodies for a large number of human proteins are available.

\section{FUTURE DIRECTIONS}

False positives or spurious interactions are estimated to occur at a high frequency in large-scale PPI studies $(90,91)$. Moreover, interactome datasets from different studies are incomplete with a large number of false negatives and are biased toward particular cellular environments and pathways that were emphasized in the screening procedure. The result is that overlap between different sets of protein interaction data that have been generated using different methods tends to be weak (90). This is even the case for the first global $\mathrm{Y} 2 \mathrm{H}$ studies that explored the interactome of yeast $S$. cerevisiae $(16,17)$. Therefore, refined PPI screening and confirmation strategies will have to be put in place to increase the validity of interaction maps. Additionally, integration with other qualitative and quantitative information will be required to validate the associations inferred by the PPI networks (92).

According to a recent estimation, about $50 \%$ of the yeast $S$. cerevisiae interactome has been explored so far, whereas the estimation for PPIs in humans is considerably lower at about $10 \%$ (93). Because of this, our knowledge of the mammalian interactome is not comparable with that of yeast. In yeast, the information on PPI networks is supported by an amassed knowledge of genetic interactions, protein expression levels, and protein localization $(13,94)$. The inherent complexity of the mammalian proteome poses another challenge. It not only encodes $\sim 5$ times more ORFs than yeast, but it is also expressed in a multitude of splice variants. Also important to note is that mammalian cells are organized in tissues and organs with diverse functions. We expect that the impressive toolbox of technologies that we present in our review will eventually reveal many aspects of the entire human interactome.

The large number of PPIs that will emerge from the combined efforts in interactive proteomics studies is expected to reveal a multitude of targets for novel therapeutics in various diseases $(95,96)$. PPIs may not be as amenable for direct therapeutic intervention as catalytic sites in enzymes, since they are not naturally evolved to accommodate small molecules. Progress is being made, however, toward the development of inhibitors that affect PPIs in pathways that are relevant in cancer $(97,98)$. In the notable case of the p53 oncoprotein, a highly potent and selective class of inhibitors has been identified that prevents the binding and targeting of $\mathrm{p} 53$ by the Mdm2 ubiquitin ligase $(97,99)$. As pointed out in this review, compounds that disrupt PPIs that are important for signaling by oncoproteins were also identified in modified Y2H screens $(28,29)$. Undoubtedly, innovative approaches in interactive proteomics are not only crucial to explore PPI interaction networks, but will also be important for identification of therapeutic targets and therefore contribute to human healthcare.

\section{ACKNOWLEDGEMENTS}

We thank Jamie Snider for critically reading the manuscript. We also thank Andrew Emili, Jack Greenblatt, Janet Rossant,

\section{GENVAULT ${ }^{\circledR}$ \\ standardizing biosample management \\ simplifying workflows \\ enabling scientific discoveries}

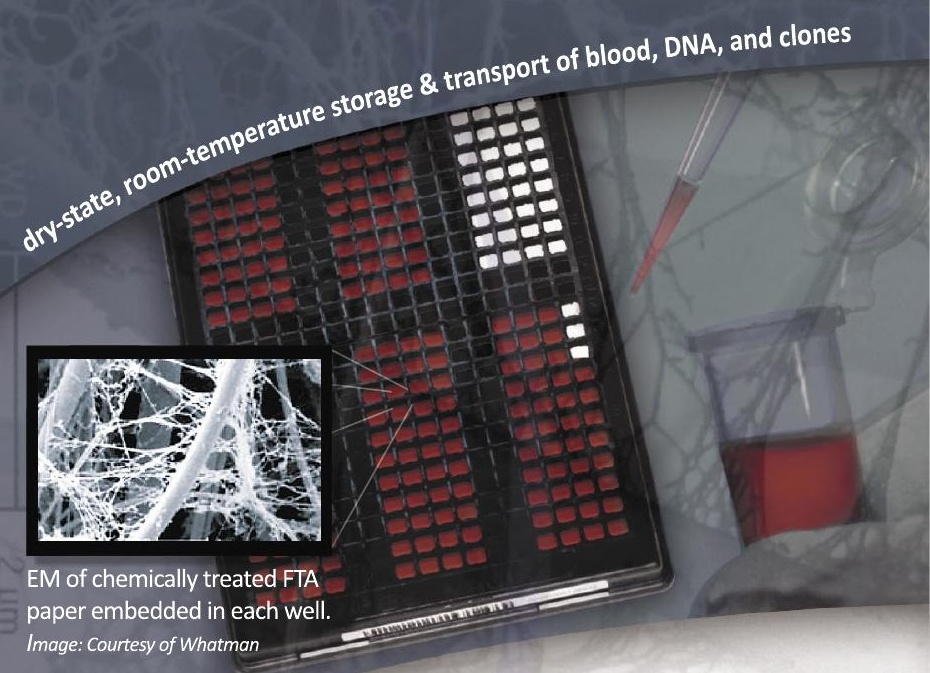

Archive

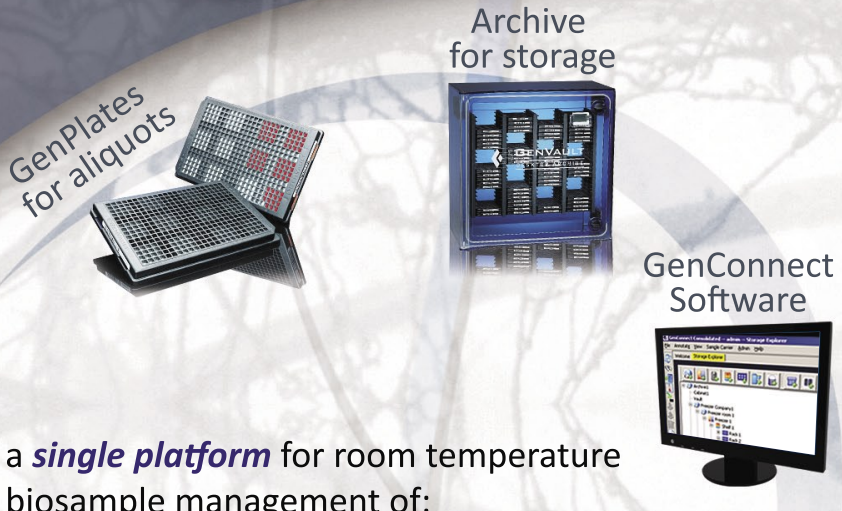

biosample management of:

primay sample collections
field collections
back-up samples
$\checkmark$ left-over samples
$\checkmark$ transport from site to site
shipping

GenSolve for DNA recovery

discovery research - biobanking identification medicine
Use recovered DNA for: PCR, WGA, genotyping microarray analysis transformations

HLA-typing and more

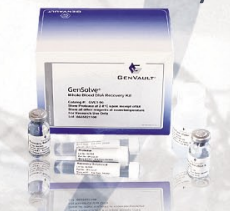
forensics - human - plant - animablic hea clinical trials diagnostics

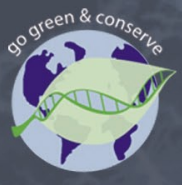


Anthony Gramiolini, and Jason Moffat for communicating their unpublished research. The Stagljar laboratory is supported by grants from the Canadian Foundation for Innovation (CFI), the Canadian Institute for Health Research (CIHR), the National Cancer Institute of Canada (NCIC), the Genome Canada and Ontario Genomics Institute, the Gebert Rüf Foundation, Genentech, and Novartis.

\section{COMPETING INTERESTS STATEMENT}

The authors declare no competing interests.

\section{REFERENCES}

1. Ng, J.H. and L.L. Ilag. 2002. Functional proteomics: separating the substance from the hype. Drug Discov. Today 7:504-505.

2. Blackstock, W.P. and M.P. Weir. 1999. Proteomics: quantitative and physical mapping of cellular proteins. Trends Biotechnol. 17:121-127.

3. Robinson, C.V., A. Sali, and W. Baumeister. 2007. The molecular sociology of the cell. Nature 450:973-982.

4. Aloy, P. and R.B. Russell. 2002. The third dimension for protein interactions and complexes. Trends Biochem. Sci. 27:633-638.

5. Kung, L.A. and M. Snyder. 2006. Proteome chips for whole-organism assays. Nat. Rev. Mol. Cell Biol. 7:617-622.

6. Bertone, P. and M. Snyder. 2005. Advances in functional protein microarray technology. FEBS J. 272:5400-5411.

7. Binz, H.K. and A. Pluckthun. 2005. Engineered proteins as specific binding reagents. Curr. Opin. Biotechnol. 16:459-469.

8. Binz, H.K., P. Amstutz, and A. Pluckthun. 2005. Engineering novel binding proteins from nonimmunoglobulin domains. Nat. Biotechnol. 23:12571268.

9. Sidhu, S.S. and S. Koide. 2007. Phage display for engineering and analyzing protein interaction interfaces. Curr. Opin. Struct. Biol. 17:481-487.

10. Goh, K.I., M.E. Cusick, D. Valle, B. Childs, M. Vidal, and A.L. Barabasi. 2007. The human disease network. Proc. Natl. Acad. Sci. USA 104:86858690 .

11. Hakes, L., J.W. Pinney, D.L. Robertson, and S.C. Lovell. 2008. Protein-protein interaction networks and biology-what's the connection? Nat. Biotechnol. 26:69-72.

12. Fields, S. and O. Song. 1989. A novel genetic system to detect protein-protein interactions. Nature 340:245-246

13. Suter, B., D. Auerbach, and I. Stagljar. 2006. Yeast-based functional genomics and proteomics technologies: the first 15 years and beyond. BioTechniques 40:625-644.

14. Parrish, J.R., K.D. Gulyas, and R.L. Finley, Jr. 2006. Yeast two-hybrid contributions to interactome mapping. Curr. Opin. Biotechnol. 17:387-393.

15. Fields, S. 2005. High-throughput two-hybrid analysis. The promise and the peril. FEBS J. 272:53915399.

16. Uetz, P., L. Giot, G. Cagney, T.A. Mansfield, R.S Judson, J.R. Knight, D. Lockshon, V. Narayan, et al. 2000. A comprehensive analysis of proteinprotein interactions in Saccharomyces cerevisiae. Nature 403:623-627.

17. Ito, T., T. Chiba, R. Ozawa, M. Yoshida, M. Hattori, and Y. Sakaki. 2001. A comprehensive two-hybrid analysis to explore the yeast protein interactome. Proc. Natl. Acad. Sci. USA 98:45694574.

18. Giot, L., J.S. Bader, C. Brouwer, A. Chaudhuri, B. Kuang, Y. Li, Y.L. Hao, C.E. Ooi, et al. 2003. A protein interaction map of Drosophila melanogaster. Science 302:1727-1736.

19. Li, S., C.M. Armstrong, N. Bertin, H. Ge, S. Milstein, M. Boxem, P.O. Vidalain, J.D. Han, et al. 2004. A map of the interactome network of the metazoan C. elegans. Science 303:540-543.

20. Stelzl, U., U. Worm, M. Lalowski, C. Haenig, F.H. Brembeck, H. Goehler, M. Stroedicke, M. Zenkner, et al. 2005. A human protein-protein interaction network: a resource for annotating the proteome. Cell 122:957-968.

21. Rual, J.F., K. Venkatesan, T. Hao, T. HirozaneKishikawa, A. Dricot, N. Li, G.F. Berriz, F.D. Gibbons, et al. 2005. Towards a proteome-scale map of the human protein-protein interaction network. Nature 437:1173-1178.

22. Oeffiner, F., C. Moch, A. Neundorf, J. Hofmann, M. Koch, and K.H. Grzeschik. 2008. Novel interaction partners of Bardet-Biedl syndrome proteins. Cell Motil. Cytoskeleton 65:143-155.

23. Lim, J., T. Hao, C. Shaw, A.J. Patel, G. Szabo, J.F. Rual, C.J. Fisk, N. Li, et al. 2006. A proteinprotein interaction network for human inherited ataxias and disorders of Purkinje cell degeneration. Cell 125:801-814

24. Colland, F., X. Jacq, V. Trouplin, C. Mougin, C. Groizeleau, A. Hamburger, A. Meil, J. Wojcik, et al. 2004. Functional proteomics mapping of a human signaling pathway. Genome Res. 14:1324-1332.

25. Fiebitz, A., L. Nyarsik, B. Haendler, Y.H. Hu, F. Wagner, S. Thamm, H. Lehrach, M. Janitz, et al. 2008. High-throughput mammalian two-hybrid screening for protein-protein interactions using transfected cell arrays. BMC Genomics 9:68.

26. Matsuzawa, S. and J.C. Reed. 2007. Yeast and mammalian two-hybrid systems for studying protein-protein interactions. Methods Mol. Biol. 383:215-225.

27. Shioda, T., S. Andriole, T. Yahata, and K.J. Isselbacher. 2000. A green fluorescent protein-reporter mammalian two-hybrid system with extrachromosomal maintenance of a prey expression plasmid: application to interaction screening. Proc. Natl. Acad. Sci. USA 97:5220-5224.

28. Yin, X., C. Giap, J.S. Lazo, and E.V. Prochownik. 2003. Low molecular weight inhibitors of Myc-Max interaction and function. Oncogene 22:6151-6159.

29. Kato-Stankiewicz, J., I. Hakimi, G. Zhi, J. Zhang, I. Serebriiskii, L. Guo, H. Edamatsu, H. Koide, et al. 2002. Inhibitors of Ras/Raf-1 interaction identified by two-hybrid screening revert Ras-dependent transformation phenotypes in human cancer cells. Proc. Natl. Acad. Sci. USA 99:14398-14403.

30. Huang, J. and S.L. Schreiber. 1997. A yeast genetic system for selecting small molecule inhibitors of protein-protein interactions in nanodroplets. Proc. Natl. Acad. Sci. USA 94:13396-13401.

31. Vidal, M., R.K. Brachmann, A. Fattaey, E. Harlow, and J.D. Boeke. 1996. Reverse two-hybrid and one-hybrid systems to detect dissociation of protein-protein and DNA-protein interactions. Proc. Natl. Acad. Sci. USA 93:10315-10320.

32. Licitra, E.J. and J.O. Liu. 1996. A three-hybrid system for detecting small ligand-protein receptor interactions. Proc. Natl. Acad. Sci. USA 93:1281712821.

33. Baker, K., D. Sengupta, G. Salazar-Jimenez, and V.W. Cornish. 2003. An optimized dexamethasone-methotrexate yeast 3-hybrid system for highthroughput screening of small molecule-protein interactions. Anal. Biochem. 315:134-137.
34. Becker, F., K. Murthi, C. Smith, J. Come, N. Costa-Roldan, C. Kaufmann, U. Hanke, C. Degenhart, et al. 2004. A three-hybrid approach to scanning the proteome for targets of small molecule kinase inhibitors. Chem. Biol. 11:211-223.

35. Stagljar, I., C. Korostensky, N. Johnsson, and S. te Heesen. 1998. A genetic system based on splitubiquitin for the analysis of interactions between membrane proteins in vivo. Proc. Natl. Acad. Sci. USA 95:5187-5192.

36. Johnsson, N. and A. Varshavsky. 1994. Split ubiquitin as a sensor of protein interactions in vivo. Proc. Natl. Acad. Sci. USA 91:10340-10344.

37. Miller, J.P., R.S. Lo, A. Ben-Hur, C. Desmarais, I. Stagljar, W.S. Noble, and S. Fields. 2005. Largescale identification of yeast integral membrane protein interactions. Proc. Natl. Acad. Sci. USA 102:12123-12128.

38. Paumi, C.M., J. Menendez, A. Arnoldo, K. Engels, K.R. Iyer, S. Thaminy, O. Georgiev, Y. Barral, et al. 2007. Mapping protein-protein interactions for the yeast $\mathrm{ABC}$ transporter Ycflp by integrated splitubiquitin membrane yeast two-hybrid analysis. Mol Cell 26:15-25.

39. Wang, B., J. Pelletier, M.J. Massaad, A. Herscovics, and G.C. Shore. 2004. The yeast split-ubiquitin membrane protein two-hybrid screen identifies BAP31 as a regulator of the turnover of endoplasmic reticulum-associated protein tyrosine phosphatase-like B. Mol. Cell. Biol. 24:2767-2778.

40. Thaminy, S., D. Auerbach, A. Arnoldo, and I. Stagljar. 2003. Identification of novel ErbB3-interacting factors using the split-ubiquitin membrane yeast two-hybrid system. Genome Res. 13:17441753.

41. Mockli, N., A. Deplazes, P.O. Hassa, Z. Zhang, M. Peter, M.O. Hottiger, I. Stagljar, and D. Auerbach. 2007. Yeast split-ubiquitin-based cytosolic screening system to detect interactions between transcriptionally active proteins. BioTechniques 42:725-730.

42. Pelletier, J.N., F.X. Campbell-Valois, and S.W. Michnick. 1998. Oligomerization domain-directed reassembly of active dihydrofolate reductase from rationally designed fragments. Proc. Natl. Acad. Sci. USA 95:12141-12146.

43. Pelletier, J.N., K.M. Arndt, A. Pluckthun, and S.W. Michnick. 1999. An in vivo library-versus-library selection of optimized protein-protein interactions. Nat. Biotechnol. 17:683-690.

44. Remy, I. and S.W. Michnick. 2001. Visualization of biochemical networks in living cells. Proc. Natl. Acad. Sci. USA 98:7678-7683.

45. Michnick, S.W., P.H. Ear, E.N. Manderson, I. Remy, and E. Stefan. 2007. Universal strategies in research and drug discovery based on proteinfragment complementation assays. Nat. Rev. Drug Discov. 6:569-582.

46. Wehr, M.C., R. Laage, U. Bolz, T.M. Fischer, S. Grunewald, S. Scheek, A. Bach, K.A. Nave, et al. 2006. Monitoring regulated protein-protein interactions using split TEV. Nat. Methods 3:985-993.

47. Barnea, G., W. Strapps, G. Herrada, Y. Berman, J. Ong, B. Kloss, R. Axel, and K.J. Lee. 2008. The genetic design of signaling cascades to record receptor activation. Proc. Natl. Acad. Sci. USA 105:64 69.

48. Eyckerman, S., A. Verhee, J.V. der Heyden, I. Lemmens, X.V. Ostade, J. Vandekerckhove, and J. Tavernier. 2001. Design and application of a cytokine-receptor-based interaction trap. Nat. Cell Biol. 3:1114-1119.

49. Baker, S.J., S.G. Rane, and E.P. Reddy. 2007 Hematopoietic cytokine receptor signaling. Oncogene 26:6724-6737.

50. Montoye, T., I. Lemmens, D. Catteeuw, S. Eyckerman, and J. Tavernier. 2005. A systematic scan of interactions with tyrosine motifs in the erythropoietin receptor using a mammalian 2-hybrid approach. Blood 105:4264-4271.

51. Uyttendaele, I., I. Lemmens, A. Verhee, A.S. De Smet, J. Vandekerckhove, D. Lavens, F. Peelman, 
and J. Tavernier. 2007. Mammalian protein-protein interaction trap (MAPPIT) analysis of STAT5, CIS, and SOCS2 interactions with the growth hormone receptor. Mol. Endocrinol. 21:2821-2831.

52. Ulrichts, P. and J. Tavernier. 2008. MAPPIT analysis of early Toll-like receptor signalling events. Immunol Lett. [7 Jan. 2007; Epub ahead of print].

53. Eyckerman, S., I. Lemmens, D. Catteeuw, A. Verhee, J. Vandekerckhove, S. Lievens, and J. Tavernier. 2005. Reverse MAPPIT: screening for protein-protein interaction modifiers in mammalian cells. Nat. Methods 2:427-433.

54. Caligiuri, M., L. Molz, Q. Liu, F. Kaplan, J.P. Xu, J.Z. Majeti, R. Ramos-Kelsey, K. Murthi, et al. 2006. MASPIT: three-hybrid trap for quantitative proteome fingerprinting of small molecule-protein interactions in mammalian cells. Chem. Biol. 13:711-722.

55. Siegel, R.M., F.K. Chan, D.A. Zacharias, R. Swofford, K.L. Holmes, R.Y. Tsien, and M.J. Lenardo. 2000. Measurement of molecular interactions in living cells by fluorescence resonance energy transfer between variants of the green fluorescent protein. Sci. STKE 2000:PL1.

56. Xu, Y., D.W. Piston, and C.H. Johnson. 1999. A bioluminescence resonance energy transfer (BRET) system: application to interacting circadian clock proteins. Proc. Natl. Acad. Sci. USA 96:151-156.

57. Boute, N., R. Jockers, and T. Issad. 2002. The use of resonance energy transfer in high-throughput screening: BRET versus FRET. Trends Pharmacol. Sci. 23:351-354.

58. Hebert, T.E., C. Gales, and R.V. Rebois. 2006. Detecting and imaging protein-protein interactions during $\mathrm{G}$ protein-mediated signal transduction in vivo and in situ by using fluorescence-based techniques. Cell Biochem. Biophys. 45:85-109.

59. Gales, C., R.V. Rebois, M. Hogue, P. Trieu, A. Breit, T.E. Hebert, and M. Bouvier. 2005. Realtime monitoring of receptor and G-protein interactions in living cells. Nat. Methods 2:177-184.

60. Bertrand, L., S. Parent, M. Caron, M. Legault, E. Joly, S. Angers, M. Bouvier, M. Brown, et al. 2002. The BRET2/arrestin assay in stable recombinant cells: a platform to screen for compounds that interact with $\mathrm{G}$ protein-coupled receptors (GPCRS). J. Recept. Signal Transduct. Res. 22:533-541.

61. Hamdan, F.F., M. Audet, P. Garneau, J. Pelletier, and M. Bouvier. 2005. High-throughput screening of $\mathrm{G}$ protein-coupled receptor antagonists using a bioluminescence resonance energy transfer 1-based beta-arrestin2 recruitment assay. J. Biomol. Screen. 10:463-475.

62. Coulon, V., M. Audet, V. Homburger, J. Bockaert, L. Fagni, M. Bouvier, and J. Perroy. 2008. Subcellular imaging of dynamic protein interactions by bioluminescence resonance energy transfer. Biophys. J. 94:1001-1009.

63. Hu, C.D., Y. Chinenov, and T.K. Kerppola. 2002. Visualization of interactions among bZIP and Rel family proteins in living cells using bimolecular fluorescence complementation. Mol. Cell 9:789-798.

64. Hu, C.D. and T.K. Kerppola. 2003. Simultaneous visualization of multiple protein interactions in living cells using multicolor fluorescence complementation analysis. Nat. Biotechnol. 21:539-545.

65. Kerppola, T.K. 2006. Visualization of molecular interactions by fluorescence complementation. Nat. Rev. Mol. Cell Biol. 7:449-456.

66. Remy, I., A. Montmarquette, and S.W. Michnick. 2004. PKB/Akt modulates TGF-beta signalling through a direct interaction with Smad3. Nat. Cell Biol. 6:358-365.

67. Stefan, E., S. Aquin, N. Berger, C.R. Landry, B. Nyfeler, M. Bouvier, and S.W. Michnick. 2007. Quantification of dynamic protein complexes using Renilla luciferase fragment complementation applied to protein kinase A activities in vivo. Proc. Natl. Acad. Sci. USA 104:16916-16921.
68. Remy, I. and S.W. Michnick. 2006. A highly sensitive protein-protein interaction assay based on Gaussia luciferase. Nat. Methods 3:977-979.

69. Paulmurugan, R., T.F. Massoud, J. Huang, and S.S. Gambhir. 2004. Molecular imaging of drugmodulated protein-protein interactions in living subjects. Cancer Res. 64:2113-2119.

70. Massoud, T.F., R. Paulmurugan, A. De, P. Ray, and S.S. Gambhir. 2007. Reporter gene imaging of protein-protein interactions in living subjects. Curr. Opin. Biotechnol. 18:31-37.

71. Soderberg, O., K.J. Leuchowius, M. KamaliMoghaddam, M. Jarvius, S. Gustafsdottir, E. Schallmeiner, M. Gullberg, J. Jarvius, et al. 2007. Proximity ligation: a specific and versatile tool for the proteomic era. Genet. Eng. 28:85-93.

72. Soderberg, O., M. Gullberg, M. Jarvius, K. Ridderstrale, K.J. Leuchowius, J. Jarvius, K. Wester, P. Hydbring, et al. 2006. Direct observation of individual endogenous protein complexes in situ by proximity ligation. Nat. Methods 3:995-1000.

73. Fredriksson, S., M. Gullberg, J. Jarvius, C. Olsson, K. Pietras, S.M. Gustafsdottir, A. Ostman, and U. Landegren. 2002. Protein detection using proximity-dependent DNA ligation assays. Nat. Biotechnol. 20:473-477.

74. Ho, Y., A. Gruhler, A. Heilbut, G.D. Bader, L. Moore, S.L. Adams, A. Millar, P. Taylor, et al. 2002. Systematic identification of protein complexes in Saccharomyces cerevisiae by mass spectrometry. Nature 415:180-183

75. Krogan, N.J., G. Cagney, H. Yu, G. Zhong, X. Guo, A. Ignatchenko, J. Li, S. Pu, et al. 2006. Global landscape of protein complexes in the yeast Saccharomyces cerevisiae. Nature 440:637-643.

76. Gavin, A.C., P. Aloy, P. Grandi, R. Krause, M. Boesche, M. Marzioch, C. Rau, L.J. Jensen, et al. 2006. Proteome survey reveals modularity of the yeast cell machinery. Nature 440:631-636.

77. Gavin, A.C., M. Bosche, R. Krause, P. Grandi, M. Marzioch, A. Bauer, J. Schultz, J.M. Rick, et al. 2002. Functional organization of the yeast proteome by systematic analysis of protein complexes. Nature 415:141-147.

78. Rigaut, G., A. Shevchenko, B. Rutz, M. Wilm, M. Mann, and B. Seraphin. 1999. A generic protein purification method for protein complex characterization and proteome exploration. Nat. Biotechnol. 17:1030-1032.

79. Puig, O., F. Caspary, G. Rigaut, B. Rutz, E. Bouveret, E. Bragado-Nilsson, M. Wilm, and B. Seraphin. 2001. The tandem affinity purification (TAP) method: a general procedure of protein complex purification. Methods 24:218-229.

80. Veraksa, A., A. Bauer, and S. Artavanis-Tsakonas. 2005. Analyzing protein complexes in Drosophila with tandem affinity purification-mass spectrometry. Dev. Dyn. 232:827-834.

81. Bouwmeester, T., A. Bauch, H. Ruffner, P.O. Angrand, G. Bergamini, K. Croughton, C. Cruciat, D. Eberhard, et al. 2004. A physical and functional map of the human TNF-alpha/NF-kappa B signal transduction pathway. Nat. Cell Biol. 6:97105.

82. Brajenovic, M., G. Joberty, B. Kuster, T. Bouwmeester, and G. Drewes. 2004. Comprehensive proteomic analysis of human Par protein complexes reveals an interconnected protein network. J. Biol. Chem. 279:12804-12811.

83. Burckstummer, T., K.L. Bennett, A. Preradovic, G. Schutze, O. Hantschel, G. Superti-Furga, and A. Bauch. 2006. An efficient tandem affinity purification procedure for interaction proteomics in mammalian cells. Nat. Methods 3:1013-1019.

84. McKinnell, I.W., J. Ishibashi, F. Le Grand, V.G. Punch, G.C. Addicks, J.F. Greenblatt, F.J. Dilworth, and M.A. Rudnicki. 2008. Pax7 activates myogenic genes by recruitment of a histone methyltransferase complex. Nat. Cell Biol. 10:77-84.
85. Aloy, P. and R.B. Russell. 2002. Potential artefacts in protein-interaction networks. FEBS Lett 530:253-254.

86. Suter, B., M.J. Fetchko, R. Imhof, C.I. Graham, I. Stoffel-Studer, C. Zbinden, M. Raghavan, L. Lopez, et al. 2007. Examining protein-protein interactions using endogenously tagged yeast arrays: the Cross-and-Capture system. Genome Res. 17:1774 1782.

87. Barrios-Rodiles, M., K.R. Brown, B. Ozdamar, R. Bose, Z. Liu, R.S. Donovan, F. Shinjo, Y. Liu, et al. 2005. High-throughput mapping of a dynamic signaling network in mammalian cells. Science 307:1621-1625.

88. Selbach, M. and M. Mann. 2006. Protein interaction screening by quantitative immunoprecipitation combined with knockdown (QUICK). Nat. Methods 3:981-983.

89. Blagoev, B., I. Kratchmarova, S.E. Ong, M. Nielsen, L.J. Foster, and M. Mann. 2003. A proteomics strategy to elucidate functional proteinprotein interactions applied to EGF signaling. Nat. Biotechnol. 21:315-318.

90. von Mering, C., R. Krause, B. Snel, M. Cornell, S.G. Oliver, S. Fields, and P. Bork. 2002. Comparative assessment of large-scale data sets of protein-protein interactions. Nature 417:399-403.

91. Huang, H., B.M. Jedynak, and J.S. Bader. 2007 Where have all the interactions gone? Estimating the coverage of two-hybrid protein interaction maps. PLoS Comput Biol 3:e214.

92. Stelzl, U. and E.E. Wanker. 2006. The value of high quality protein-protein interaction networks for systems biology. Curr. Opin. Chem. Biol. 10:551-558.

93. Hart, G.T., A.K. Ramani, and E.M. Marcotte. 2006. How complete are current yeast and human protein-interaction networks? Genome Biol. 7:120.

94. Boone, C., H. Bussey, and B.J. Andrews. 2007. Exploring genetic interactions and networks with yeast. Nat. Rev. Genet. 8:437-449.

95. Keskin, O., A. Gursoy, B. Ma, and R. Nussinov. 2007. Towards drugs targeting multiple proteins in a systems biology approach. Curr. Top. Med. Chem. 7:943-951

96. Ruffner, H., A. Bauer, and T. Bouwmeester. 2007. Human protein-protein interaction networks and the value for drug discovery. Drug Discov. Today 12:709-716.

97. Fry, D.C. and L.T. Vassilev. 2005. Targeting protein-protein interactions for cancer therapy. J. Mol. Med. 83:955-963.

98. Arkin, M. 2005. Protein-protein interactions and cancer: small molecules going in for the kill. Curr. Opin. Chem. Biol. 9:317-324.

99. Vassilev, L.T., B.T. Vu, B. Graves, D. Carvajal, F. Podlaski, Z. Filipovic, N. Kong, U. Kammlott et al. 2004. In vivo activation of the p53 pathway by small-molecule antagonists of MDM2. Science 303:844-848

Addresss correspondence to Igor Stagljar, Department of Biochemistry and Department of Molecular Genetics, Terrence Donnelly Centre for Cellular and Biomolecular Research (dCCBR), University of Toronto, 160 College Street, Toronto ON M5S 3E1 Canada. e-mail: igor. stagljar@utoronto.ca

To purchase reprints of this article, contact: Reprints@BioTechniques.com 